Bull. Austral. Math. Soc.

VOL. $70(2004)$ [7-15]

\title{
ON GENERALISED MIXED CO-QUASI-VARIATIONAL INEQUALITIES WITH NONCOMPACT VALUED MAPPINGS
}

\author{
Rais Ahmad, Qamrul Hasan Ansari and Syed Shakaib Irfan
}

In this paper, we consider generalised mixed co-quasi-variational inequalities with noncompact valued mappings and propose an iterative algorithm for computing their approximate solutions. We prove that the approximate solutions obtained by the proposed algorithm converge to the exact solution of our co-quasi-variational inequality. Some special cases are also discussed.

\section{InTRODUCTION AND FORMULATION}

The projection iterative method is one of the most important and useful methods for finding the approximate solutions of fixed point problems, and variational and quasi-variational inequality problems; See for example $[5,6,8,9,10,11,13,14]$ and references therein. In most of the papers appearing in the literature on this topic, the metric projection operators, as in Hilbert spaces, are used. But it is impossible to use the metric projection operator in the setting of Banach spaces because these operators are not nonexpansive. Recently, Takahashi and Kim [15] used the sunny nonexpansive retraction to set up an iterative scheme for finding a fixed point of a nonexpansive and nonself mapping in Banach spaces. Inspired by the work of Takahashi and Kim [15], Alber and Yao [2] used a sunny nonexpansive retraction to construct the projection iterative method for finding the approximate solutions of a class of multivalued quasi-variational inequalities in Banach spaces. They gave the name co-quasi-variational inequality for a quasi-variational inequality in Banach spaces and presented an iterative algorithm. They also proved several convergence results for approximate solutions obtained by their algorithms and in particular several existence results were obtained. Recently, Chang [4] also studied the existence and convergence of solutions of the Mann and Ishikawa iterative processes for a class of variational inclusions with accretive type mappings in Banach spaces. The mathematical approach in [4] is quite different from the one used by Alber and Yao [2].

Received June 24, 2004

The second author is grateful to the Department of Mathematical Sciences, King Fahd University of Petroleum \& Minerals, Dhahran, Saudi Arabia for providing excellent research facilities.

Copyright Clearance Centre, Inc. Serial-fee code: 0004-9727/04 \$A2.00+0.00. 
Let $B$ be a real Banach space with norm $\|\|,. B^{*}$ its topological conjugate space with norm $\|.\|_{*}$ and $\langle x, f\rangle$ be a pairing between $x \in B$ and $f \in B^{*}$. Given single-valued mappings $f, g, p, G: B \rightarrow B$ and multivalued mappings $M, S, T, K: B \rightarrow 2^{B}$ such that $\forall x \in B, K(x)$ is a nonempty, closed and convex set, we consider the following generalised mixed co-quasi-variational inequality problem :

$$
\left\{\begin{array}{l}
\text { Find } x \in B, u \in M(x), v \in S(x) \text { and } w \in T(x) \\
\text { such that } G(x) \in K(x) \text { and } \\
\langle p(u)-(f(v)-g(w)), J(z-G(x))\rangle \geqslant 0, \quad \forall z \in K(x),
\end{array}\right.
$$

where $J: B \rightarrow B^{*}$ is the normalised duality mapping and $2^{B}$ is the family of all nonempty subsets of $B$.

The nonlinear operator $J: B \rightarrow B^{*}$ is called normalised duality mapping if

$$
\|J x\|_{*}=\|x\| \text { and }\langle x, J(x)\rangle=\|x\|^{2}, \forall x \in B .
$$

For further detail of the duality mapping $J$, we refer to [1].

Let us see some special cases of generalised mixed co-quasi-variational inequality problems.

SPECIAL CASES:

(i) If $p \equiv 0, g$ and $M$ are identity mappings and $T$ is single-valued mapping, then generalised mixed co-quasi-variational inequality problem reduces to the problem of finding $x \in B$ and $v \in S(x)$ such that $G(x) \in K(x)$ and

$$
\langle T(x)-f(v), J(z-G(x))\rangle \geqslant 0, \quad \forall z \in K(x),
$$

A problem similar to (1.1) is recently considered and studied by Alber and Yao [2].

(ii) If $B$ is a Hilbert space, $f, g$ and $M$ are identity mappings, $G(x)=p(x)$ and $K(x)=K \forall x \in B$ then generalised mixed co-quasi-variational inequality problem becomes the following generalised variational inequality problem considered and studied by Verma [16]:

$$
\left\{\begin{array}{l}
\text { Find } x \in B, v \in S(x) \text { and } w \in T(x) \text { such that } \\
\langle p(x)-(v-w), z-p(x)\rangle \geqslant 0, \quad \forall z \in K
\end{array}\right.
$$

It is clear that the generalised mixed co-quasi-variational inequality problem includes many kinds of quasi-variational inequality problems, variational inequality problems and complementarity problems as special cases, such as $[8,13,16,17]$. 
In this paper, we extend the approach of Alber and Yao [2] to a more general and unified problem called the generalised mixed co-quasi-variational inequality problem. We suggest and analyse an iterative algorithm to compute the approximate solutions of the generalised mixed co-quasi-variational inequality problem with noncompact valued mappings. We also prove convergence result for the approximate solutions obtained by the proposed algorithm.

\section{Preliminaries}

We recall that the uniform convexity of the Banach space $B$ means that for any given $\varepsilon>0$ there exists $\delta>0$ such that $\forall x, y \in B,\|x\| \leqslant 1,\|y\| \leqslant 1,\|x-y\|=\varepsilon$ ensure the following inequality

$$
\|x+y\| \leqslant 2(1-\delta)
$$

The function

$$
\delta_{B}(\varepsilon)=\inf \left\{1-\frac{\|x+y\|}{2}:\|x\|=1,\|y\|=1,\|x-y\|=\varepsilon\right\}
$$

is called the modulus of the convexity of the space $B$.

The uniform smoothness of the space $B$ means that for any given $\varepsilon>0$, there exists $\delta>0$ such that

$$
\frac{\|x+y\|+\|x-y\|}{2}-1 \leqslant \varepsilon\|y\|
$$

holds. The function

$$
\rho_{B}(t)=\sup \left\{\frac{\|x+y\|+\|x-y\|}{2}-1:\|x\|=1,\|y\|=t\right\}
$$

is called the modulus of the smoothness of the space $B$.

We remark that the space $B$ is uniformly convex if and only if $\delta_{B}(\varepsilon)>0$ for all $\varepsilon>0$, and it is uniformly smooth if and only if $\lim _{t \rightarrow 0} t^{-1} \rho_{B}(t)=0$. The following inequalities will be used in the proof of our main result.

Proposition 2.1. ([1]) Let $B$ be a uniformly smooth Banach space and $J$ be a normalised duality mapping from $B$ to $B^{*}$. Then, $\forall x, y \in B$, we have

(i) $\|x+y\|^{2} \leqslant\|x\|^{2}+2\langle y, J(x+y)\rangle$,

(ii) $\langle x-y, J(x)-J(y)\rangle \leqslant 2 d^{2} \rho_{B}(4\|x-y\| / d)$, where $d=\sqrt{\left(\|x\|^{2}+\|y\|^{2}\right) / 2}$.

We next recall the following definitions.

Definition 2.1: A mapping $A: B \rightarrow B$ is said to be

(i) strongly accretive if there exists a constant $\gamma>0$ such that

$$
\langle A(x)-A(y), J(x-y)\rangle \geqslant \gamma\|x-y\|^{2}, \forall x, y \in B
$$


(ii) Lipschitz continuous if there exists a positive constant $\beta$ such that

$$
\|A(x)-A(y)\| \leqslant \beta\|x-y\|, \forall x, y \in B .
$$

Definition 2.2: A multivalued map $S: B \rightarrow C B(B)$ is said to be $\mathcal{H}$-Lipschitz continuous if there exists a constant $h>0$ such that

$$
\mathcal{H}(S(x), S(y)) \leqslant h\|x-y\|, \forall x, y \in B .
$$

where $\mathcal{H}(.,$.$) is the Hausdorff metric and C B(B)$ is the family of all nonempty closed bounded subsets of $B$.

Definition 2.3: $([3,7])$ Let $B$ be a real Banach space and $\Omega$ a nonempty closed convex subset of $B$. A mapping $Q_{\Omega}: B \rightarrow \Omega$ is said to be

(i) retraction on $\Omega$ if $Q_{\Omega}^{2}=Q_{\Omega}$;

(ii) nonexpansive retraction if in addition

$$
\left\|Q_{\Omega} x-Q_{\Omega} y\right\| \leqslant\|x-y\|, \forall x, y \in B ;
$$

(iii) sunny retraction if $\forall x \in B$,

$$
Q_{\Omega}\left(Q_{\Omega} x+t\left(x-Q_{\Omega}\right)\right)=Q_{\Omega} x, \forall t \in \mathbb{R} .
$$

The following characterisation of a sunny nonexpansive retraction mapping can be found in $[3,7]$.

PROPOSITION 2.2. $Q_{\Omega}$ is a sunny nonexpansive retraction if and only if $\forall x \in B$ and $\forall y \in \Omega$

$$
\left\langle x-Q_{\Omega} x, J\left(Q_{\Omega} x-y\right)\right\rangle \geqslant 0 .
$$

From the above Proposition 2.2, we have the following retraction shift equality.

Proposition 2.3. Let $B$ be a Banach space, $\Omega$ a nonempty closed and convex subset of $B$ and $m: B \rightarrow B$ a mapping. Then $\forall x \in B$, we have

$$
Q_{\Omega+m(x)} x=m(x)+Q_{\Omega}(x-m(x)) .
$$

\section{An Iterative Algorithm}

In this section, we first derive some characterisations of solutions of generalised mixed co-quasi-variational inequality problem.

We mention the following characterisation theorem for the solution of generalised mixed co-quasi-variational inequality problem which can be easily proved by using Proposition 2.2 and the argument of [8, Theorem 3.1] and [1, Theorem 8.1].

THEOREM 3.1. Let $B$ be a Banach space, $f, g, p, G: B \rightarrow B$ single-valued mappings, $M, S, T: B \rightarrow C B(B)$ mappings and $K: B \rightarrow 2^{B}$ multivalued mappings such that $\forall x \in B, K(x)$ is a nonempty, closed and convex subset. Then the following statements are equivalent: 
(i) The set of elements $(x, u, v, w)$ such that $x \in B, u \in M(x), v \in S(x)$ and $w \in T(x)$ is a solution of generalised mixed co-quasi-variational inequality problem.

(ii) $x \in B, u \in M(x), v \in S(x), w \in T(x)$ and

$$
G(x)=Q_{K(x)}[G(x)-\tau(p(u)-(f(v)-g(w)))] \text { for any } \tau>0 .
$$

Combining Proposition 2.3 and Theorem 3.1, we obtain the following result.

THEOREM 3.2. Let $B$ be a real Banach space, $X$ a nonempty, closed and convex subset of $B$. Let $f, g, p, m, G: B \rightarrow B$ be single-valued mappings, and $M, S, T: B$ $\rightarrow C B(B)$ mappings and $K: B \rightarrow 2^{B}$ multivalued mappings such that $\forall x$ $\in B, K(x)=m(x)+X$. Then the set of elements $(x, u, v, w)$ such that $x \in B, u$ $\in M(x), v \in S(x)$ and $w \in T(x)$ is a solution of generalised mixed co-quasi-variational inequality problem if and only if $x=x-G(x)+m(x)+Q_{X}[G(x)-\tau(p(u)-(f(v)-g(w)))-m(x)]$, for any $\tau>0$.

To compute the approximate solutions of generalised mixed co-quasi-variational inequality problem, we propose the following iterative algorithm.

Algorithm 3.1. Let $K(x)=m(x)+X$, where $X$ is a nonempty, closed and convex subset of $B$ and $\tau>0$ be fixed. Let $f, g, p, m, G: B \rightarrow B$ be single-valued mappings and $S, M, T: B \rightarrow C B(B)$ be multivalued mappings. For given $u_{0} \in M\left(x_{0}\right), v_{0} \in S\left(x_{0}\right)$ and $w_{0} \in T\left(x_{0}\right)$, let

$$
x_{1}=x_{0}-G\left(x_{0}\right)+m\left(x_{0}\right)+Q_{X}\left[G\left(x_{0}\right)-\tau\left(p\left(u_{0}\right)-\left(f\left(v_{0}\right)-g\left(w_{0}\right)\right)\right)-m\left(x_{0}\right)\right] .
$$

Since $u_{0} \in M\left(x_{0}\right) \in C B(B), v_{0} \in S\left(x_{0}\right) \in C B(B), w_{0} \in T\left(x_{0}\right) \in C B(B)$, by Nadler [12], there exist $u_{1} \in M\left(x_{1}\right), v_{1} \in S\left(x_{1}\right), w_{1} \in T\left(x_{1}\right)$ such that

$$
\begin{aligned}
\left\|u_{0}-u_{1}\right\| & \leqslant(1+1) \mathcal{H}\left(M\left(x_{0}\right), M\left(x_{1}\right)\right), \\
\left\|v_{0}-v_{1}\right\| & \leqslant(1+1) \mathcal{H}\left(S\left(x_{0}\right), S\left(x_{1}\right)\right), \\
\left\|w_{0}-w_{1}\right\| & \leqslant(1+1) \mathcal{H}\left(T\left(x_{0}\right), T\left(x_{1}\right)\right) .
\end{aligned}
$$

Let

$$
x_{2}=x_{1}-G\left(x_{1}\right)+m\left(x_{1}\right)+Q_{X}\left[G\left(x_{1}\right)-\tau\left(p\left(u_{1}\right)-\left(f\left(v_{1}\right)-g\left(w_{1}\right)\right)\right)-m\left(x_{1}\right)\right] .
$$

By induction, we can obtain the sequences $\left\{x_{n}\right\},\left\{u_{n}\right\},\left\{v_{n}\right\}$ and $\left\{w_{n}\right\}$ as

$$
x_{n+1}=x_{n}-G\left(x_{n}\right)+m\left(x_{n}\right)+Q_{X}\left[G\left(x_{n}\right)-\tau\left(p\left(u_{n}\right)-\left(f\left(v_{n}\right)-g\left(w_{n}\right)\right)\right)-m\left(x_{n}\right)\right],
$$




$$
\begin{gathered}
u_{n} \in M\left(x_{n}\right), \quad\left\|u_{n}-u_{n+1}\right\| \leqslant\left(1+(n+1)^{-1}\right) \mathcal{H}\left(M\left(x_{n}\right), M\left(x_{n+1}\right)\right), \\
v_{n} \in S\left(x_{n}\right), \quad\left\|v_{n}-v_{n+1}\right\| \leqslant\left(1+(n+1)^{-1}\right) \mathcal{H}\left(S\left(x_{n}\right), S\left(x_{n+1}\right)\right), \\
w_{n} \in T\left(x_{n}\right), \quad\left\|w_{n}-w_{n+1}\right\| \leqslant\left(1+(n+1)^{-1}\right) \mathcal{H}\left(T\left(x_{n}\right), T\left(x_{n+1}\right)\right),
\end{gathered}
$$

$n=0,1,2,3, \ldots$

Finally, we prove that the approximate solutions obtained by Algorithm 3.1 converge to the exact solution of generalised mixed co-quasi-variational inequality problem. We also prove the existence of a solution of generalised mixed co-quasi-variational inequality problem.

THEOREM 3.3. Let $B$ be a real uniformly smooth Banach space with the module of smoothness $\tau_{B}(t) \leqslant D t^{2}$ for some $D>0$. Let $X$ be a nonempty, closed and convex subset of $B, f, g, p, m, G: B \rightarrow B$ single-valued mappings and $M, S, T: B \rightarrow C B(B)$ and $K: B \rightarrow 2^{B}$ multivalued mappings such that $\forall x \in B, K(x)=m(x)+X$. Suppose that the following conditions are satisfied:

(i) $f, g$ and $p$ are Lipschitz continuous with corresponding constants $\xi, r$ and $\sigma$, respectively.

(ii) $G$ is both strongly accretive with constant $\gamma$ and Lipschitz continuous with constant $\delta$.

(iii) $M, S, T$ are $\mathcal{H}$-Lipschitz continuous with corresponding constants $s, h$ and $d$, respectively.

(iv) $m$ is Lipschitz continuous with constant $\theta$.

(v) $0<2\left(1-2 \gamma+64 D \delta^{2}\right)^{1 / 2}+2 \theta+\tau \sigma s+[1+\tau(\xi h-r d)]<1$.

Then there exists a set of elements $(x, u, v, w)$ such that $x \in B, u \in M(x), v \in S(x)$ and $w \in T(x)$ which is a solution of generalised mixed co-quasi-variational inequality problem and $x_{n} \rightarrow x, u_{n} \rightarrow u, v_{n} \rightarrow v, w_{n} \rightarrow w$ as $n \rightarrow \infty$, where $\left\{x_{n}\right\},\left\{u_{n}\right\},\left\{v_{n}\right\}$ and $\left\{w_{n}\right\}$ are the sequences obtained by Algorithm (3.1).

ProOF: By the iterative scheme 3.1 and Proposition 2.3, we have

$$
\begin{aligned}
& \left\|x_{n+1}-x_{n}\right\|=\| x_{n}-G\left(x_{n}\right)+m\left(x_{n}\right)+Q_{X}\left[G\left(x_{n}\right)-\tau\left(p\left(u_{n}\right)-\left(f\left(v_{n}\right)\right.\right.\right. \\
& \left.\left.\left.\quad-g\left(w_{n}\right)\right)\right)-m\left(x_{n}\right)\right]-\left(x_{n-1}-G\left(x_{n-1}\right)\right)+m\left(x_{n-1}\right) \\
& \quad-Q_{X}\left[G\left(x_{n-1}\right)-\tau\left(p\left(u_{n-1}\right)-\left(f\left(v_{n-1}\right)-g\left(w_{n-1}\right)\right)\right)-m\left(x_{n-1}\right)\right] \| \\
& \leqslant\left\|x_{n}-x_{n-1}-\left(G\left(x_{n}\right)-G\left(x_{n-1}\right)\right)\right\|+2\left\|m\left(x_{n}\right)-m\left(x_{n-1}\right)\right\| \\
& \quad+\left\|x_{n}-x_{n-1}-\left(G\left(x_{n}\right)-G\left(x_{n-1}\right)\right)\right\|+\tau\left\|p\left(u_{n}\right)-p\left(u_{n-1}\right)\right\| \\
& \quad+\left\|x_{n}-x_{n-1}+\tau\left(f\left(v_{n}\right)-f\left(v_{n-1}\right)\right)-\tau\left(g\left(w_{n}\right)-g\left(w_{n-1}\right)\right)\right\| \\
& =2\left\|x_{n}-x_{n-1}-\left(G\left(x_{n}\right)-G\left(x_{n-1}\right)\right)\right\|+2\left\|m\left(x_{n}\right)-m\left(x_{n-1}\right)\right\|
\end{aligned}
$$




$$
\begin{aligned}
& +\tau\left\|p\left(u_{n}\right)-p\left(u_{n-1}\right)\right\|+\| x_{n}-x_{n-1}+\tau\left(f\left(v_{n}\right)-f\left(v_{n-1}\right)\right) \\
& -\tau\left(g\left(w_{n}\right)-g\left(w_{n-1}\right)\right) \|
\end{aligned}
$$

By Proposition 2.1, we have (see, for example, the proof of [2, Theorem 3])

$$
\left\|x_{n}-x_{n-1}-\left(G\left(x_{n}\right)-G\left(x_{n-1}\right)\right)\right\|^{2} \leqslant\left(1-2 \gamma+64 D \delta^{2}\right)\left\|x_{n}-x_{n-1}\right\|^{2} .
$$

It is clear that

$$
\left\|m\left(x_{n}\right)-m\left(x_{n-1}\right)\right\| \leqslant \theta\left\|x_{n}-x_{n-1}\right\| .
$$

Since $M, S$ and $T$ are $\mathcal{H}$-Lipschitz continuous, and $f, g$ and $p$ are Lipschitz continuous, we have

$$
\begin{gathered}
\left\|p\left(u_{n}\right)-p\left(u_{n-1}\right)\right\| \leqslant \sigma\left\|u_{n}-u_{n-1}\right\| \leqslant \sigma s\left(1+\frac{1}{n}\right)\left\|x_{n}-x_{n-1}\right\|, \\
\left\|f\left(v_{n}\right)-f\left(v_{n-1}\right)\right\| \leqslant \xi\left\|v_{n}-v_{n-1}\right\| \leqslant \xi h\left(1+\frac{1}{n}\right)\left\|x_{n}-x_{n-1}\right\|, \\
\left\|g\left(w_{n}\right)-g\left(w_{n-1}\right)\right\| \leqslant r\left\|w_{n}-w_{n-1}\right\| \leqslant r d\left(1+\frac{1}{n}\right)\left\|x_{n}-x_{n-1}\right\| .
\end{gathered}
$$

From (3.6)-(3.7), it follows that

$$
\begin{aligned}
& \left\|x_{n}-x_{n-1}+\tau\left(f\left(v_{n}\right)-f\left(v_{n-1}\right)\right)-\tau\left(g\left(w_{n}\right)-g\left(w_{n-1}\right)\right)\right\| \\
& \quad \leqslant\left\|x_{n}-x_{n-1}\right\|+\tau\left\|f\left(v_{n}\right)-f\left(v_{n-1}\right)\right\|-\tau\left\|g\left(w_{n}\right)-g\left(w_{n-1}\right)\right\| \\
& \leqslant\left\|x_{n}-x_{n-1}\right\|+\tau \xi h(1+1 / n)\left\|x_{n}-x_{n-1}\right\|-\tau r d(1+1 / n)\left\|x_{n}-x_{n-1}\right\| \\
& \quad=[1+\tau(1+1 / n)(\xi h-r d)]\left\|x_{n}-x_{n-1}\right\| .
\end{aligned}
$$

Combining (3.2)-(3.5) and (3.8), it follows that

$$
\left\|x_{n+1}-x_{n}\right\| \leqslant t_{n}\left\|x_{n}-x_{n-1}\right\|
$$

where $t_{n}=2\left(1-2 \gamma+64 D \delta^{2}\right)^{1 / 2}+2 \theta+\tau \sigma s(1+(1 / n))+[1+\tau(1+1 / n)(\xi h-r d)]$.

Let $t=2\left(1-2 \gamma+64 D \delta^{2}\right)^{1 / 2}+2 \theta+\tau \sigma s+[1+\tau(\xi h-r d)]$. Then $t_{n} \rightarrow t$ as $n \rightarrow \infty$. It follows from $(v)$ that $t<1$. Hence $t_{n}<1$ for $n$ sufficiently large. Consequently $\left\{x_{n}\right\}$ is a Cauchy sequence in $B$. Since $B$ is complete, we can let $x_{n} \rightarrow x \in B$. Now we prove that $u_{n} \rightarrow u \in M(x), v_{n} \rightarrow v \in S(x)$ and $w_{n} \rightarrow w \in T(x)$. In fact, it follows from Algorithm 3.1 that

$$
\begin{aligned}
\left\|u_{n}-u_{n-1}\right\| & \leqslant\left(1+\frac{1}{n}\right) s\left\|x_{n}-x_{n-1}\right\| \\
\left\|v_{n}-v_{n-1}\right\| & \leqslant\left(1+\frac{1}{n}\right) h\left\|x_{n}-x_{n-1}\right\| \\
\left\|w_{n}-w_{n-1}\right\| & \leqslant\left(1+\frac{1}{n}\right) d\left\|x_{n}-x_{n-1}\right\|
\end{aligned}
$$


which implies that $\left\{u_{n}\right\},\left\{v_{n}\right\}$ and $\left\{w_{n}\right\}$ are also Cauchy sequences in $B$. Again, since $B$ is complete, we can let $u_{n} \rightarrow u, v_{n} \rightarrow v, w_{n} \rightarrow w$ as $n \rightarrow \infty$. Since $Q_{X}, G, f, g, p, M, S, T$ and $m$ are continuous in $B$, we have

$$
x=x-G(x)+m(x)+Q_{X}[G(x)-\tau(p(u)-(f(v)-g(w)))-m(x)] .
$$

Further, we have

$$
\begin{aligned}
d(v, S(x)) & =\inf \{\|v-y\|: y \in S(x)\} \\
& \leqslant\left\|v-v_{n}\right\|+d\left(v_{n}, S(x)\right) \\
& \leqslant\left\|v-v_{n}\right\|+\mathcal{H}\left(S\left(x_{n}\right), S(x)\right) \\
& \leqslant\left\|v-v_{n}\right\|+h\left\|x_{n}-x\right\| \rightarrow 0 \text { as } n \rightarrow \infty
\end{aligned}
$$

Hence $v \in S(x)$. Similarly we can prove that $u \in M(x), w \in T(x)$. The result then follows from Theorem 3.2.

REMARK 3.1. As a special case of Algorithm 3.1, we can easily derive an iterative algorithm, similar to the algorithm proposed in [2], for finding the approximation solutions of problem (1.1).

\section{REFERENCES}

[1] Y. Alber, 'Metric and generalized projection operators in Banach Spaces; properties and applications', in Theory and Applications of Nonlinear Operators of Monotone and Accretive Type, (A. Kartsatos, Editor) (Marcel Dekker, New York, 1996), pp. 15-50.

[2] Y. Alber and J.C. Yao, 'Algorithm for generalized multi-valued co-variational inequalities in Banach Spaces', Funct. Differ. Equ. 7 (2000), 5-13.

[3] Y. Banyamini and J. Lindenstrauss, Geometric nonlinear functional analysis, I, American Mathematical Society Colloquium Publications 48 (American Mathematical Society, Providence, R.I., 2000).

[4] S.S. Chang, 'On the Mann and Ishikawa iterative approximation of solution to variational inclusions with accretive mappings', Comput. Math. Appl. 37 (1999), 17-24.

[5] M. Chipot, Elements of nonlinear analysis (Birkhauser Verlag, Berlin, 2000).

[6] R. Glowinski, J.L. Lions and R. Tremolieres, Numerical analysis of variational inequalities (North-Holland, Amsterdam, 1981).

[7] K. Goebel and S. Reich, Uniform convexity, hyperbolic geometry and nonexpansive mappings (Marcel Dekker, New York, N.Y., 1984).

[8] J.S. Guo and J.C. Yao, 'Extension of strongly nonlinear quasivariational inequalities', Appl. Math. Lett. 5 (1992), 35-38.

[9] G. Isac, Complementarity problems (Springer-Verlag, Berlin, 1992).

[10] G. Isac, Topological methods in complementarity theory (Kluwer Academic Publishers, Dordrecht, Boston, London, 2000).

[11] D. Kinderlehrer and G. Stampacchia, An introduction to variational inequalities and their applications (Academic Press, New York, 1980). 
[12] S.B. Nadler, Jr., 'Multi-valued contraction mappings', Pacific J. Math. 30 (1969), 475-488.

[13] A.H. Siddiqi and Q.H. Ansari, 'Strongly nonlinear quasivariational inequalities', J. Math. Anal. Appl. 149 (1990), 444-450.

[14] A.H. Siddiqi and Q.H. Ansari, 'General strongly nonlinear variational inequalities', $J$. Math. Anal. Appl. 166 (1992), 386-392.

[15] W. Takahashi and G.-E. Kim, 'Strong convergence of approximates to fixed points of nonexpansive nonself-mappings in Banach spaces', Nonlinear Anal. 32 (1998), 447-454.

[16] R.U. Verma, 'On generalized variational inequalities involving relaxed Lipschitz and relaxed monotone operators', J. Math. Anal. Appl. 213 (1997), 387-392.

[17] J.C. Yao, 'Applications of variational inequalities to nonlinear analysis', Appl. Math. Lett. 4 (1991), 89-92.

Department of Mathematics

Aligarh Muslim University

Aligarh 202002

India

e-mail: raisain@lycos.com

Department of Mathematics

Aligarh Muslim University

Aligarh 202002

India

e-mail: shakaib11@rediffmail.corn
Department of Mathematical Sciences

King Fahd University of Petroleum \& Minerals

P.O. Box 1169

Dhahran 31261

Saudi Arabiaa

e-mail: qhansari@kfupm.edu.sa

Second author is on leave from

Department of Mathematics

Aligarh Muslim University

Aligarh 202002

India

e-mail: qhansari@sancharnet.in 